\title{
ABSOLUTE CONTINUITY AND HYPONORMAL OPERATORS
}

\author{
C.R. PUTNAM \\ Department of Mathematics \\ Purdue University \\ West Lafayette, Indiana 47907 \\ (Received July 18, 1980)
}

ABSTRACT. Let $T$ be a completely hyponormal operator, with the rectangular representation $T=A+i B$, on a separable Hilbert space. If 0 is not an eigenvalue of $T^{*}$ then $T$ also has a polar factorization $T=U P$, with $U$ unitary. It is known that A, B and $U$ are all absolutely continuous operators. Conversely, given an arbitrary absolutely continuous selfadjoint A or unitary $U$, it is shown that there exists a corresponding completely hyponormal operator as above. It is then shown that these ideas can be used to establish certain known absolute continuity properties of various unitary operators by an appeal to a lemma in which, in one interpretation, a given unitary operator is regarded as a polar factor of some completely hyponormal operator. The unitary operators in question are chosen from a number of sources: the F. and M. Riesz theorem, dissipative and certain mixing transformations in ergodic theory, unitary dilation theory, and minimal normal extensions of subnormal contractions.

KEY WORDS AND PHRASES. Selfadjoint operators, unitary operators, hyponormal operators, ergodic theory, unitary dilations, subnormal operators.

1980 MATHEMATICS SUBJECT CLASSIFICATION CODES: Primary: 47B15, 47B20; Secondary: 26A46, 28AT0, 47A35, 47A55, 47B47.

1. INTRODUCTION.

Let $T$ be a bounded operator, with the rectangular decomposition 
$T=A+i B$, on a complex, separable Hilbert space $H$, and denote $i$ ts spectrum and point spectrum by $\sigma(T)$ and $\sigma_{p}(T)$ respectively. Then $T$ is said to be hyponormal if

$$
T^{\star} T-T^{*}=D \geqq 0, \text { equivalently, } A B-B A=-i C(C=12 D \geqq 0),
$$

and completely hyponormal if, in addition, $T$ has no normal part, that is, if there is no nontrivial reducing space of $T$ on which it is normal. (For a recent survey of hyponormal operators, see Clancey [1].) If $T$ is hyponormal then the sets $\sigma(A)$ and $\sigma(B)$ are just the real projections of $\sigma(T)$ onto the real and imaginary axes; see Putnam [2], p. 46. It is known that if $T$ is completely hyponormal then $A=\operatorname{Re}(T)$, as well as $B=\operatorname{Im}(T)=\operatorname{Re}(-i T)$, is absolutely continuous, that is, if $A$ has the spectral resolution

$$
A=\int t d E_{t},
$$

then $\left\|E_{t} x\right\|^{2}$ is an absolutely continuous function of $t$ for every $x$ in $H$; see [2], p. 42.

Since (1.1) holds if $T$ is replaced by its translate $T$ - $z I$, it is clear that if $T$ is hyponormal then $\sigma_{p}(T) \subset \sigma_{p}\left(T^{*}\right)$, and it is not hard to show that if $T$ is completely hyponormal then $\sigma_{p}(T)$ is empty. Also, if $T$ is completely hyponormal then it has a (unique) polar factorization

$$
\left.T=U P, \quad U=\text { unitary (hence } P=\left(T^{*} T\right)^{\frac{1}{2}}\right),
$$

if and only if $0 \notin \sigma_{\mathrm{p}}\left(\mathrm{T}^{*}\right)$; see the discussion in Putnam [3], pp. 419-420. If $T$ is completely hyponormal with the factorization (1.3) then $U$ is also absolutely continuous; see [2], p. 21 and Lemma 4 of [4]. Thus, if $U$ has the spectral resolution

$$
U=\int_{0}^{2 \pi} e^{i t} d G_{t}\left(\int_{0}^{2 \pi}=\int_{0}^{2 \pi \infty 0}\right),
$$

then $\left\|G_{t} x\right\|^{2}$ is an absolutely continuous function of $t$ for every $x$ in $H$. 
A converse of the above assertions concerning absolute continuity also holds, namely :

(*) Every bounded absolutely continuous selfadjoint operator $A$ is the real part of some completely hyponormal operator.

(**) Every absolutely continuous unitary operator $U$ is the unitary factor in the polar factorization (1.3) of some completely hyponormal operator.

That there exists some nonnormal hyponormal operator $T$ for which $A=\operatorname{Re}(T)$ was shown by Kato [5], and that $T$ can even be constructed so that it is completely hyponormal can be deduced from Kato's results. A direct proof of (*) will be given below however.

Let $E$ be any bounded subset of $(-\infty, \infty)$ having positive measure and let $T_{0}=A_{0}+i B_{0}$, where, for $x$ in $L^{2}(E),\left(A_{0} x\right)(t)=t x(t)$ and $\left(B_{0} x\right)(t)=-(i \pi)^{-1} \int_{E}(s-t)^{-1} x(s) d s$, the integral being a cauchy principal value. It is easily verified that ${ }_{0} B_{0}-B_{0} A_{0}=-i C_{0}$, where $C_{0} x=\pi^{-1}(x, 1) 1$ and 1 denotes the identity function on $L^{2}(E)$. Since the vectors $\left\{A_{0}^{n_{1}}\right\}$, $n=0,1,2, \ldots$, span $L^{2}(E)$, it is clear that $T_{0}$ is completely hyponormal on $L^{2}(E)$; see, e.g., Clancey and Putnam [6], p. 452 .

It is now easy to prove (*). According to spectral multiplicity theory applied to absolutely continuous operators, $A$ is unitarily equivalent to, and here will simply be assumed to be equal to, the following canonical representation. There exist disjoint Borel sets $\left\{E_{n}\right\}$ on the real line, when $n \in \alpha$ and $\alpha$ is a nonempty subset of $\left\{1,2, \ldots, \kappa_{0}\right\}$, and where each $E_{n}$ has positive measure and $\bigcup_{n \in \alpha} E_{n}$ is a bounded set. The operator $A$ is a direct sum $A=\sum \oplus A_{n}$ on $H=\Sigma \oplus H_{n} \quad(n \in \alpha)$, where each $H_{n}$ is the direct sum of $n$ copies of $L^{2}\left(E_{n}\right)$ and $\left(A_{n} x_{n}\right)(t)=t x_{n}(t)$ for $x_{n}$ in $H_{n}$. Since each $A_{n}$ is the real part of the direct sum, $T_{n}$, of $n$ copies of $T_{0}=A_{0}+i B_{0}$ on the corresponding space $H_{n}$, then $A=R e(T)$, where $T=\Sigma \oplus T_{n}$ on $H=\Sigma \oplus H_{n} \quad(n \in \alpha)$. That T is completely hyponormal is a consequence of the properties of $T_{0}$ on $L^{2}\left(E_{n}\right)$ 
as described earlier, and so $(*)$ is proved.

Although the selfcommutator $T * T-T T *=D$ of the above operator $T$ need not be of trace class, it is clear that such a $T$ could be constructed. This is apparent from the fact that $T_{n}$ could as well be replaced by $\left.\sum_{k=1}^{n} \oplus^{\left(A_{0}+i t\right.} k^{B}\right)^{\prime}$, where the $t_{n k}(n \in \alpha, k=1, \ldots, n)$ are any positive constants whose total sum is finite.

In order to prove $(* *)$, first choose $S_{0}$ to be any completely hyponormal operator on the Hilbert space $\mathrm{H}$ with a polar factorization $\mathrm{S}_{0}=\mathrm{U}_{0} \mathrm{P}_{0}$, where $\mathrm{U}_{0}$ is unitary with simple multiplicity on the entire unit circle $|z|=1$. For example, let $\left\{\lambda_{n}\right\}, n=0, \pm 1, \pm 2, \ldots$, be any bounded, strictly increasing sequence of numbers satisfying, say, $\underset{n \rightarrow-\infty}{\lim _{n}} \lambda_{n}=1$ and $\underset{n \rightarrow \infty}{\lim } \lambda_{n}=2$. If $H$ is the $\ell^{2}$ bilateral sequence Hilbert space, let $A=\left(a_{i j}\right)$ and $B=\left(b_{i j}\right)$ be the doubly infinite matrices defined by $a_{i j}=\delta_{i j} \lambda_{j}$ and $b_{i j}=\delta_{i j} \lambda_{j-1}$. Then $A=$ WBW $^{*}$ where $\mathrm{W}$ is the unitary bilateral shift $\mathbf{x}=\left(\ldots, \mathrm{x}_{-1},\left(\mathrm{x}_{0}\right), \mathrm{x}_{1}, \ldots\right) \rightarrow$ $W x=\left(\ldots, x_{0},\left(x_{1}\right), x_{2}, \ldots\right)$, the parentheses referring to the 0 position. If $S_{0}=U_{0} A^{\frac{1}{2}}$, where $U_{0}=W^{\star}$, then $S_{0} s_{0}-S_{0} S_{0} *=A-B=D \geqq 0$, and, since o\& $\sigma_{p}(D), s_{0}$ is completely hyponormal. Also, $s_{0}$ is nonsingular, that is, $0 \notin \sigma\left(S_{0}\right)$. In fact, $\sigma\left(s_{0}\right)=\{z: 1 \leqq|z| \leqq \sqrt{2}\} ;$ see, e.g., Th. 1 and Th. 9 of [3]. In addition, $U_{0}$ has the desired properties. (See also section 3 below.) Next, let $U_{0}$ have the spectral resolution $U_{0}=\int_{0}^{2 \pi} e^{i t} \mathrm{dG}_{t}^{0}$, and, for any Borel set $\beta \subset[0,2 \pi]$ of positive measure, consider the unitary operator $G^{0}(\beta) U_{O}$ on the Hilbert space $G^{0}(\beta) H$. (Note that $G^{0}(\beta) \neq 0$ if and only if $B$ has positive measure; see also (3.2) below.) If $S_{0}(\beta)=G^{0}(\beta) U_{0}\left[G^{0}(\beta) S_{0}{ }^{*} S_{0} G^{0}(\beta)\right]^{\frac{1}{2}}$, then a simple calculation shows that $S_{O}(\beta)$ is hyponormal on $G^{0}(\beta) H$. In addition, $S_{0}(\beta)$ is completely hyponormal on $G^{0}(\beta) H_{;}$see Lemma 8 of Putnam [7], pp. 326-327. From here on, the proof of $(* *)$ is essentially identical to that of (*) but where unitary operators now play the role formerly played by 
selfadjoint ones. Also, corresponding to a similar argument above, the completely hyponormal operator $T$ of $(* *)$ can be chosen so that $T * T-T T^{*}$ is of trace class.

Absolutely continuous selfadjoint and unitary operators occur in various connections. The results of $(*)$ and $(* *)$ indicate that the study of such operators can be reduced, at least in principle, to the study of the real part, or of the unitary factor in the polar factorization, of an appropriate completely hyponormal operator. In the sections below this (somewhat tendentious) point of view will be exploited to investigate certain unitary operators arising in the following situations: bilateral shifts and their role in the F. and M. Riesz theorem and in dissipative and certain mixing transformations in ergodic theory; unitary dilations of contractions; minimal normal extensions of subnormal contractions.

2. A LEMMA.

The following lemma (I) asserts some absolute continuity properties of operators effecting the unitary equivalence of two selfadjoint operators having a positive difference. As will be noted below, it concerns the unitary operators of polar factorizations of certain corresponding completely hyponormal operators. It will play a key role in later applications.

LEMMA (I) Let $J$ and $D$ be bounded, selfadjoint operators on a Hilbert space $H$ and suppose that $D \geqq 0$ and $\neq 0$. Let $U$ be unitary with the spectral resolution (1.4) and suppose that

$$
J-U U^{*}=D
$$

(i) If

$H$ is the least space reducing both $J$ and $U$ and containing the range of $D$,

then $U$ is absolutely continuous. 
(ii) If $\beta$ is any Borel subset of $[0,2 \pi]$ for which $G(\beta)=I$ (and whether or not (2.2) is satisfied) then

$$
2 \pi\|D\| \leqq d(J)|\beta| \text {, where } d(J)=\max \sigma(J)-\min \sigma(J) .
$$

Here, $\max \sigma(J)$ and $\min \sigma(J)$ denote the maximum and minimum points of $\sigma(J)$ and $|\beta|$ denotes linear Lebesgue measure of $\beta$. (The proof of a slightly different formulation of (I) is given in Putnam [8] under the added hypothesis that $O \notin \sigma_{p}(D)$, in which case, condition (2.2) is automatically assured.) Note that $\|D\| \leqq d(J)$ and that, in (ii), if there exists a $J$ for which $d(J)=\|D\|>0$, or even if there exists a sequence $J_{1}, J_{2}, \ldots$, and a corresponding sequence $D_{1}, D_{2}, \ldots$, for which $d\left(J_{n}\right)=1 \mathrm{im}\left\|D_{n}\right\|>0$ (finite), then $|\beta|=0$ whenever $\beta$ is any Borel subset of $[0,2 \pi]$ for which $G(\beta)=0$.

In order to prove (i), note that $J$ can be replaced by $J-k I$ for any real constant $k$ and hence there is no loss of generality in supposing that $\mathrm{J} \geqq$ const $>0$. Then, in particular, $T=U J^{\frac{1}{2}}$ satisfies (1.1) by virtue of (2.1), so that $T$ is hyponormal. That $T$ is completely hyponormal is a consequence of (2.2) and, as noted in section $I$ above, $U$ is absolutely continuous.

The proof of (ii) essentially has been given in [8]. An alternate proof can be obtained from an inequality concerning hyponormal operators derived in [4] . In fact, if $T$ is any completely hyponormal operator with factorization (1.3) and if, for $0 \leqq t<2 \pi$,

$$
\alpha_{t}=\sigma(T) \cap\{z: \arg z=t\} \text { and } M(t)=\int_{\alpha_{t}} \operatorname{rdr},
$$

then, for any Borel set $\beta$ of $[0,2 \pi)$,

$$
\pi\|G(\beta) D G(\beta)\| \leqq \int_{\beta} M(t) d t .
$$

See Theorem 1 of [4], where the additional hypothesis that $T$ be completely hyponormal is imposed. This restriction is not necessary, however. In fact, if $T$ is normal, $D=0$ and $(2.5)$ becomes trivial. If $T$ is not normal, then one 
need only split off its normal part and then apply the result in [4] to the remaining completely hyponormal part of $T$. (Note that the spectrum of the restriction of $T$ to a reducing subspace is a subset of the spectrum of $T$ on the entire space.) If $T=U J^{\frac{1}{2}}$ as defined above, then clearly $\sigma(T)$ is a subset of the annulus centered at the origin with inner radius $(\min \sigma(J))^{\frac{1}{2}}$ and outer radius $(\max \sigma(J))^{\frac{1}{2}}$. Consequently, $M(t) \leqq \frac{1}{2}(\max \sigma(J)-\min \sigma(J))$ and (2.3) follows from (2.5).

\section{BILATERAL SHIFTS}

Let $U$ be unitary on $H$ and let $K \neq 0$ be a subspace of $H$ for which

$$
K \perp U^{n} K \text { for } n= \pm 1, \pm 2, \ldots, \text { and } H=\sum_{-\infty}^{\infty} \oplus U^{n} K
$$

Then $U$ is a bilateral shift on $\mathrm{H}$ of (constant) multiplicity = dim $\mathrm{K}$. (A space $K$ satisfying the first part of (3.1) is called a wandering subspace of $U$. ) If a bilateral shift $U$ has the spectral resolution (1.4) then it is known that for any Borel subset $\beta$ of $[0,2 \pi]$,

$$
G(\beta)=0 \text { if and only if }|\beta|=0
$$

This fact can be derived from $(I)$ as follows. Let $\left\{\lambda_{n}\right\}, n=0, \pm 1, \pm 2, \ldots$, be any bounded, nondecreasing sequence of real numbers. For any $x$ in $H$ one has the (unique) representation $x=\sum x_{n}$ where $x_{n} \in U^{n} K$. Let the selfadjoint operator $J$ on $H$ be defined by $J x=\sum \lambda_{n} x_{n}$. It is seen that $U J^{*} x=\sum \lambda_{n-1} x_{n}$, and so (2.1) holds with $\mathrm{Dx}=\sum\left(\lambda_{n}-\lambda_{n-1}\right) x_{n} \cdot \operatorname{clearly}, D \geqq 0$. If now \{ $\mathrm{d}$, is strictly increasing, then $O \& \sigma_{p}(D)$, and hence (2.2) holds. The first half of (3.2) now follows from (i) of (I). Next, if the $\left\{\lambda_{n}\right\}$ sequence is chosen so that, say, $\lambda_{n}=0$ for $n \leqq 0$ and $\lambda_{n}=1$ for $n \geqq 1$, then the corresponding operators $J$ and $D$ satisfy $d(J)=\|D\|=1$ and so the second part of (3.2) follows from (ii) of (I).

The F. and M. Riesz theorem [9] furnishes an interesting instance of how 
bilateral shifts can arise. Let $\lambda$ be normalized linear Lebesgue measure and $\mu$ any nonzero, finite, complex Borel measure on the unit circle $c:|z|=1$. The F. and M. Riesz theorem is the assertion that

$$
\int_{C} z^{n} d \mu=0 \text { for } n=1,2, \ldots
$$

implies

$$
|\mu| \ll \lambda \ll|\mu|
$$

that is, $|\mu|$ and $\lambda$ are equivalent measures on $C$. If $U$ is the unitary operator on $L^{2}(|\mu|)$ of multiplication by $z$ and if $M$ is the subspace of $L^{2}(|\mu|)$ spanned by $\left\{z, z^{2}, \ldots\right\}$ then

$$
M=\sum_{n=0}^{\infty} \oplus U^{n} K,
$$

where $K$ is the one-dimensional wandering subspace $M \ominus U M$ and $I^{2}(|\mu|)=$ $\sum_{-\infty}^{\infty} \oplus U^{n} \mathrm{k}$. See Sarason [10], pp. 14-18, Sarason [11], and putnam [8]. It turns out ([8]) that (3.4) is simply an interpretation of (3.2).

It may be noted that the representation (3.5) is a form of what is usually called the Wold decomposition for isometries. (See Sz. Nagy and Foias, [12], pp. 3, 51 for a statement of the theorem and some of its history. Perhaps it should be referred to as the von Neumann-Wold-Halmos decomposition.)

Another instance where the bilateral shift occurs comes from ergodic theory. Here, one has a system $(X, S, m, \phi)$ consisting of a measure space, $X$, of points $x$ and a measure, $m$, defined on some $\sigma$-algebra, $s$, of subsets of $x$, where $0<m(X) \leqq \infty$. In addition, $\phi$ is here assumed to be an invertible measure preserving transformation of $x$ onto itself. (See e.g., Brown [13], Halmos [14].) Let $U$ be the unitary operator defined by

$$
U: f(x) \rightarrow f(\phi(x)) \text { on } L^{2}(x, d m) \text {. }
$$

In case there exists a set $A \subset S$ for which $m(A)>0$ and the images 
$A_{n}=\phi^{n}(A) \quad(n=0, \pm 1, \pm 2, \ldots)$ are disjoint and $x=\bigcup_{\infty}^{\infty} A_{n}$, then $A$ is a generating set of $x$ and $\phi$ is said to be dissipative; see Halmos [14], p. 11, Hopf [15], p. 46. In case $\phi$ is dissipative, then $m(x)=\infty$ and 0 is a bilateral shift. In fact, if $K=I^{2}(A, d m)$ then $K$ is a wandering subspace of $U$, so that (3.1) holds with $H=L^{2}(x, d m)$.

In case $m(x)<\infty$, the unitary operatory $U$ of $(3.6)$ has 1 in its point spectrum, the characteristic function of $\mathrm{x}$ being an eigenvector. However, even in this case it may happen that $U$ is a bilateral shift on the orthogonal complement $L^{2}(x, \mathrm{dm}) \ominus R$, where $R$ denotes the eigenspace corresponding to the eigenvalue 1. This happens, for instance, if $(x, s, m, \phi)$ is a Kolmogorov (or $\mathrm{K}-$ ) system, in which case $\phi$ is a special type of mixing transformation called a K-automorphism. For the definitions of these concepts, see Arnold and Arez [16], pp. 32-35, 153-157, Brown [13], p. 39, problem 25. Then $R$ is the space of constants in $L^{2}(x, d m)$ and the space $L^{2}(x, d m) \ominus R$ admits a decomposition of the form (3.1) for some wandering subspace $K$. Incidentally, in this case, $U$ may be a bilateral shift of infinite multiplicity; cf. [16], pp. 156-157.

\section{UNITARY DILATIONS}

Let $T$ be a contraction $(\|T\| \leqq 1)$ on the Hilbert space $H$. It is a wellknown result of B. Sz.-Nagy that there exists a Hilbert space $\mathrm{K} \supset \mathrm{H}$ and a unitary operator $U$ on $K$ with the property that

$$
\mathrm{T}^{\mathrm{n}}=\mathrm{PU}^{\mathrm{n}} \mid \mathrm{H}, \quad \mathrm{n}=0,1,2, \ldots
$$

where $P$ is the orthogonal projection $P: K \rightarrow H$. Such a unitary $U$ is called a unitary dilation of $T$. (It is easily verified that also $T^{\star n}=\mathrm{PU}^{\star n} \mid \mathrm{H}$ for $\mathrm{n}=1,2, \ldots$. .) The minimal unitary dilation, which is known to be unique, is then obtained by restricting $U$ to the least subspace of $K$ which reduces $U$ and contains H. (A brief discussion of unitary dilation theory, its history, applications, and references, can be found in Halmos [17]. For an extensive 
treatment of the subject see Sz.-Nagy and Foias, [12] and the more recent survey article of Douglas [18].)

Suppose now that the contraction $T$ is completely nonunitary (c.n.u.), so that there is no nontrivial reducing space of $T$ on which its restriction is unitary. Thus, either $T * T \neq I$ or $T T^{*} \neq I$. If the minimal unitary dilation, $U$, of such a contraction has the spectral resolution (1.4) then it is an important property of $U$ that it satisfies (3.2), a result first established by B. Sz.-NagY and C. Foias, [19]. In general, however, $U$ is itself not a bilateral shift, although (3.2) does imply that it always contains such a shift. More precisely, there is a subspace of $K$ which reduces $U$ and on which it is a bilateral shift. This is clear if one notes that (3.2) implies that $U$ has a spectral multiplicity of at least 1 at every point of the unit circle $|z|=1$.

It will be shown below how the assertion (3.2) for the minimal unitary dilation of a c.n.u. contraction can be deduced from the lemma (I) of section 2 . First, a few preliminaries will be discussed. For the given contraction, $T$, the so-called defect operators are defined by $D_{T}=\left(I-T^{\star} T\right)^{\frac{1}{2}}$ and $D_{T^{*}}=\left(I-T T^{*}\right)^{\frac{1}{2}}$, and it is fairly easy to show that

$$
\mathrm{TD}_{\mathrm{T}}=\mathrm{D}_{\mathrm{T} \star^{\mathrm{T}}} \text { and } \mathrm{T}^{\star} \mathrm{D}_{\mathrm{T}}=\mathrm{D}_{\mathrm{T}^{\mathrm{T}}} \text {; }
$$

see, e.g., [18], p. 165. Let

$$
\mathrm{K}_{1}=\ldots \oplus \mathrm{H} \oplus(\mathrm{H}) \oplus \mathrm{H} \oplus \ldots
$$

where the parentheses refer to the 0 position. If one defines the operator matrix

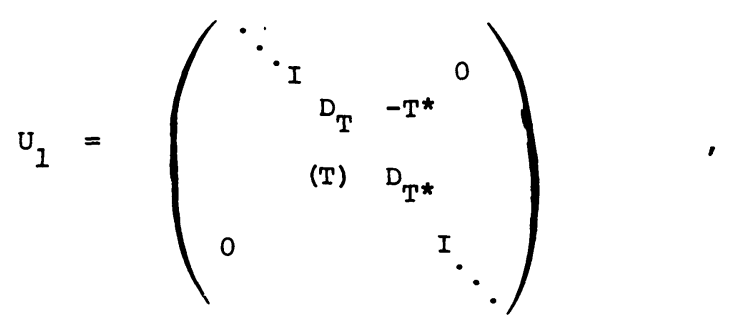


where $(T)$ refers to the $(0,0)$ element, then $U_{1}$ is readily verified to be a unitary dilation of $T$ on the space $k_{1}$ of (4.3). This explicit construction of a unitary dilation is due to J. J. Schaffer [20]. In order to obtain the minimal unitary dilation one need only restrict $U_{1}$ to the least subspace of $\mathrm{K}_{1}$ which reduces $\mathrm{U}_{1}$ and contains $\mathrm{H}$.

That even $U_{1}$ is absolutely continuous (that is, the "if" part of (3.2) if $\left\{G_{t}\right\}$ refers now to the spectral family of $U_{1}$ ) will be obtained as an application of (i) of (I) of section 2. Let $\left\{\lambda_{n}\right\}, n=0, \pm 1, \pm 2, \ldots$, be a real sequence satisfying

$$
\ldots \geqq \lambda_{-2} \geqq \lambda_{-1} \geqq \lambda_{0} \geqq \lambda_{1} \geqq \lambda_{2} \geqq \ldots \text { and }\left|\lambda_{n}\right| \leqq \text { const }<\infty \text {, }
$$

and define the selfadjoint diagonal operator matrix $J$ on $K_{1}$ of (4.3) by

$$
J=\ldots \oplus \lambda_{-I} I \oplus\left(\lambda_{0} I\right) \oplus \lambda_{I} I \oplus \ldots
$$

A straightforward calculation using (4.2) and the (crucial) equality $\lambda_{0}=\lambda_{1}$ of (4.5) shows that

$$
J-U U^{*}=D=\ldots \oplus\left(\lambda_{-1}-\lambda_{0}\right) I \oplus(0) \oplus\left(\lambda_{1}-\lambda_{2}\right) I \oplus \ldots
$$

Also, in view of $(4.5), D \geqq 0$.

By choosing the $\lambda_{n}^{\prime}$ 's so that $\lambda_{0}=\lambda_{1}$ and $\lambda_{n-1}>\lambda_{n}$ for $n \neq 1$, it is clear from (4.7) that if $M$ is the least subspace of $K_{1}$ which reduces both $J$ and $U$ and contains the range of $D$ then $M^{\perp} \subset \mathrm{H}$. Since $\mathrm{M}^{\perp}$ reduces $U$ and since $T$ is c.n.u., then $M^{\perp}=0$, that is, $M=K_{1}$. Thus, (2.2) holds with the $H$ occurring there now replaced by $K_{1}$, and so $U_{1}$ of (4.4) is absolutely continuous. This implies, of course, that its restriction to any nontrivial reducing subspace, and, in particular, the minimal unitary dilation, must also be absolutely continuous.

Moreover, corresponding to a similar argument in section 3 , one may choose $\lambda_{n}=1$ if $k \leqq 1$ and $\lambda_{n}=0$ if $n \geqq 2$. Then (4.5) holds and if the 
corresponding $J$ is defined by (4.6), with $D$ given by (4.7), then clearly $l=d(J)=\|D\|$. It follows from (ii) of the lemma (I) that $|B|=0$ whenever $G(\beta)=0, \quad \beta$ being a Borel subset of $[0,2 \pi]$ and $\left\{G_{t}\right\}$ being the spectral family of $U_{1}$ of (4.4). However, the corresponding assertion for the minimal unitary dilation does not immediately follow.

Thus, so far, (3.2) has been established when $\left\{G_{t}\right\}$ is the spectral family of $U_{1}$ but only the "if" part of (3.2) has been proved when $\left\{G_{t}\right\}$ is the spectral family of the minimal unitary dilation $U$ ( $T$ being a c.n.u. contraction). It is not hard to establish the "only if" part too however. In fact, let $\mathrm{k}$ be the subspace of $k_{1}$ of those vectors $x=\ldots+x_{-1}+\left(x_{0}\right)+x_{1}+\ldots$, where $x_{0}$ is in $H$ and $x_{n}$ is in the closure of $D_{T} H$ for $n \leqq-1$ and in the closure of $\mathrm{D}_{\mathrm{T}^{*}}{ }^{\mathrm{H}}$ for $\mathrm{n} \geqq 1$. Then $\mathrm{K}$ is the least subspace of $\mathrm{K}_{1}$ reducing $U$ and containing $H$, so that $U=U_{1} \mid K$ is the minimal unitary dilation of $T$. '(See [12], pp. 17-18.) Since $T$ is c.n.u., either $D_{T} \neq 0$ or $D_{T *} \neq 0$. Suppose, for definiteness, that $D_{T} \neq 0$. (If $D_{T}=0$ and $D_{T^{*}} \neq 0$, the treatment is similar.) Note that $J$ of (4.6) leaves $K$ invariant and $J$ is now restricted to this space. If one chooses the sequence of (4.5) so that $\lambda_{n}=1$ for $n \leqq-1$ and $\lambda_{n}=0$ for $n \geqq 0$, then clearly $d(J)=\|D\|=1$. Hence, by (ii) of the lemma (I), if $\beta$ is any Borel subset of $[0,2 \pi]$ and if the minimal unitary dilation $U=U_{1} \mid K$ has the spectral resolution (1.4), then $|\beta|=0$ whenever $G(\beta)=0$. This establishes (3.2) where the minimal unitary dilation has the spectral resolution (1.4).

Incidentally, it can be shown that if $U$ on $K$ is the minimal unitary dilation of a c.n.u. contraction $T$ on $H(C K)$ then $L=$ closure of $(U-T) H$ and $L^{*}=$ closure of $\left(U^{*}-T^{*}\right) H$ are wandering subspaces of $U$ and

$$
K=\ldots \oplus U^{* 2} L^{*} \oplus U^{*} L^{*} \oplus L^{*} \oplus H \oplus L \oplus U L \oplus U^{2} L \oplus \ldots .
$$

See [12], p. 57. The argument applied above to $U_{1}$ on $K_{1}$ and then to its restriction $U=U_{1} K$ can now be applied directly to $U$ on $K$ of $(4.8)$ in 
order to establish, again, the relation $(3.2)$ for $U$.

\section{SUBNORMAL CONTRACTIONS}

Let $T$ be subnormal on $H$ so that there exists a Hilbert space $K^{\prime} \supset$ H and a normal operator $\mathbf{N}$ on $\mathrm{K}^{\prime}$ such that $\mathrm{NH} \subset \mathrm{H}$ and $\mathbf{T}=\mathrm{N} / \mathrm{H}$. Then $\mathbf{N}$ is a normal extension of $T$. It is known that there is a unique minimal normal extension, and $\mathrm{N}$ will henceforth be assumed to be of this type. A subnormal $T$ is said to be completely subnormal if $T$ has no normal part. It may be noted, incidentally, that the subnormal operators constitute a proper subset of the set of hyponormal operators. (Concerning the basic properties of subnormal operators, see [17]. A more elaborate treatment of subnormal operator theory can be found in the recent tract of Conway and olin [21].

Suppose now that $T$ is a completely subnormal contraction on $H$ with the minimal normal extension $N$ on $K^{\prime}$ and with the minimal unitary dilation $U$ of $K$. (The space $H$ is regarded as a subspace of $K$ and of $K^{\prime}$. ) Let $P^{\prime}$ and $P$ denote the orthogonal projections $\mathrm{P}^{\prime}: \mathrm{K}^{\prime} \rightarrow \mathrm{H}$ and $\mathrm{P}: \mathrm{K} \rightarrow \mathrm{H}$, and let $\mathrm{N}$ and $U$ have the spectral resolutions $N=\int \mathrm{zdE}_{\mathrm{z}}\left(=\int_{|\mathrm{z}| \leqq l} \mathrm{zdE} \mathrm{z}_{\mathrm{z}}\right)$ and $U=\int_{C} z d_{z}(C=\{z:|z|=1\})$. Then, for $x$ in $H$ and $n=1,2, \ldots$ one has $T^{n} x=N^{n} X=P U^{n} x$ and $T^{*} n_{X}=P^{\prime} N^{*} n_{X}=P U^{*}{ }_{x}$. Hence, if $f$ is analytic on $|z|<1$ and continuous on $|z| \leqq 1$, then, for $x$ in $H, \int f(z) d E_{z} x=$ $P \int_{C} f(z) d G_{z} x$ and $P^{\prime} \int \bar{f}(z) d E_{z} x=P \int_{C} \bar{f}(z) d G_{z} x$. Consequently, if $h$ is any (real) function harmonic in $|z|<1$ and continuous on $|z| \leqq 1$, then

$$
P^{\prime} \int_{|z| \leqq 1} \operatorname{hdE} x=P \int_{C} \operatorname{hag}_{z} x, x \text { in } H
$$

Let $B$ be any closed subset of the unit circle $C$ and let $\phi$ be a real continuous function on $C$ such that $\phi=1$ on $B$ and $0 \leqq \phi<1$ on $C-B$. (Clearly, such functions exist.) Then it is well-known that there exists an $h$ as described above (which can be given explicitly by the Poisson integral) for 
which $\mathrm{h}=\phi$ on $C$. In view of (5.1), one has

$$
P^{\prime}\left(\int_{|z|<1} h d E_{z}+\int_{C} \phi d E_{z}\right) x=P \int_{C} \phi d G_{z} x \text { for } x \text { in } H \text {. }
$$

From basic max-min properties of harmonic functions it is clear that $0 \leqq h \leqq 1$ in $|z|<1$, and so, on forming inner products of the vectors in (5.2) with $x$, one obtains $\int_{C} \phi d\left\|E_{z} x\right\|^{2} \leqq \int_{C} \phi d\left\|G_{z} x\right\|^{2}$. On choosing a sequence of functions $\phi=\phi_{n}(n=1,2, \ldots)$ converging to the characteristic function of $\beta$ one obtains

$$
\|E(\beta) x\| \leqq\|G(\beta) x\|, \quad x \text { in } H .
$$

Since (5.3) holds for an arbitrary closed set $\beta$ on $C$ it clearly holds also for any Borel set $\beta$ on $C$. Let $|\beta|$ denote Lebesgue arc length measure on c. In particular, if $|\beta|=0$, then, in view of the absolute continuity of the minimal unitary dilation of a c.n.u. contraction (cf. section 4 above), $G(\beta)=0$ (on $K)$, and so $E(\beta) P^{\prime}=0$ (on $\left.K^{\prime}\right)$. Hence $0=N^{* n} E(\beta) P^{\prime}=E(\beta) N^{* n} P^{\prime}$ for $n=0,1,2, \ldots$. Since $N$ is the minimal normal extension of $T, K^{\prime}$ is spanned by the vectors $\left\{\mathrm{N}^{\star} \mathrm{Nx}: \mathrm{x} \in \mathrm{H}, \mathrm{n}=0,1,2, \ldots\right\}$, and so $E(\beta)=0$.

Thus, if $\beta$ is any Borel subset of $C$ for which $|\beta|=0$ then also $E(B)=0$. In other words, the minimal normal extension of a completely subnormal contraction is absolutely continuous on the unit circle $|z|=1$. Other proofs of this last assertion can be found in Conway and olin [21], p. 35, olin [22], and Putnam [23].

ACKNOWLEDGMENT. This work was supported by a National Science Foundation research grant.

\section{REFERENCES}

1. CLANCEY, K. Seminormal operators, Lecture notes in mathematics, $\underline{742}$, Springer-Verlag, 1979.

2. PUTNAM, C. R. Commutation properties of Hilbert space operators and related topics, Ergebnisse der Math., 36, Springer, 1967. 
3. PUTNAM, C. R. Spectra of polar factors of hyponormal operators, Trans. Amer. Math. Soc., 188 (1974), 419-428.

4. PUTNAM, C. R. A polar area inequality for hyponormal spectra, Jour. Operator Theory (to appear).

5. KATO, T. Smooth operators and commutators, Studia Math., 31 (1968), 535-546.

6. CLANCEY, K. F. and PUTNAM, C. R. The spectra of hyponormal integral operators, Comm. Math. Helv., 46 (1971), 451-456.

7. PUTNAM, C. R. An inequality for the area of hyponormal spectra, Math. Zeits., $116(1970), 323-330$.

8. PUTNAM, C. R. The F. and M. Riesz theorem revisited, Integral Equations and Operator Theory (to appear).

9. RIESZ, F. and RIESZ, M. Über die Randwerte einer analytischen Funktionen, Quat. Cong. des Math. Scand. Stockholm (1916), 27-44.

10. SARASON, D. E. Invariant subspaces, Topics in operator theory, Edit. C. Pearcy, Math. Survey, No. 13, Amer. Math. Soc., 1974, $2^{\text {nd }}$ printing, with addendum, 1979, 1-47.

11. SARASON, D. E. The theorem of F. and M. Riesz on the absolute continuity of analytic measures, unpublished note circulated c. 1963.

12. SZ.-NAGY, B. and FOIAS, C. Harmonic analysis of operators on Hilbert space, North Holland Pub. Co., American Elsevier Pub. Co., Inc., Akadémiai Kiad6, 1970.

13. BROWN, J. R. Ergodic theory and topological dynamics, Academic Press, 1976.

14. HALMOS, P. R. Lectures on ergodic theory, Chelsea Pub. Co., 1956.

15. HOPF, E. Ergodentheorie, Chelsea Pub. Co., 1948.

16. ARNOLD, V. I. and AVEZ, A. Ergodic problems of classical mechanics, พ. A. Benjamin, Inc., 1968.

17. HALMOS, P. R. A Hilbert space problem book, Van Nostrand Co., Inc., 1967.

18. DOOGLAS, R. G. Canonical models, Topics in operator theory, Edit. C. Pearcy, Math. Survey, No. 13, Amer. Math. Soc., 1974, $2^{\text {nd }}$ printing, with addendum, 1979, 161-218.

19. SZ.-NAGY, B. and FOIAS, C. Sur les contractions de l'espace de Hilbert. IV, Acta Sci. Math. (Szeged) 21 (1960), 251-259.

20. SCHAFFER, J. J. On unitary dilations of contractions, Proc. Amer. Math. Soc., $6(1955), 322$.

21. CONWAY, J. and OLIN, R. F. A functional calculus for subnormal operators II, Memoirs Amer. Math. Soc., 10, No. 184, May, 1977.

22. OIIN, R. F. Functional relationships between a subnormal operator and its minimal normal extension, Thesis, Indiana Univ., 1975.

23. PUTNAM, C. R. Peak sets and subnormal operators, Ill. Jour. Math., 21 (1977), 388-394. 


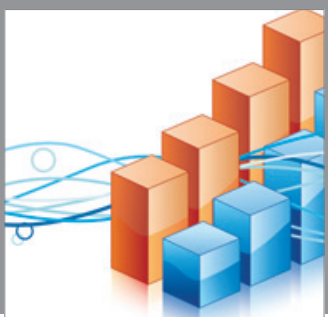

Advances in

Operations Research

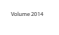

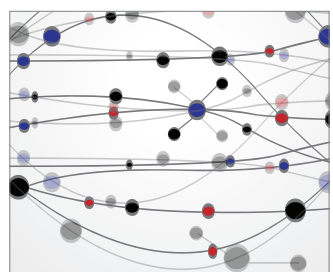

\section{The Scientific} World Journal
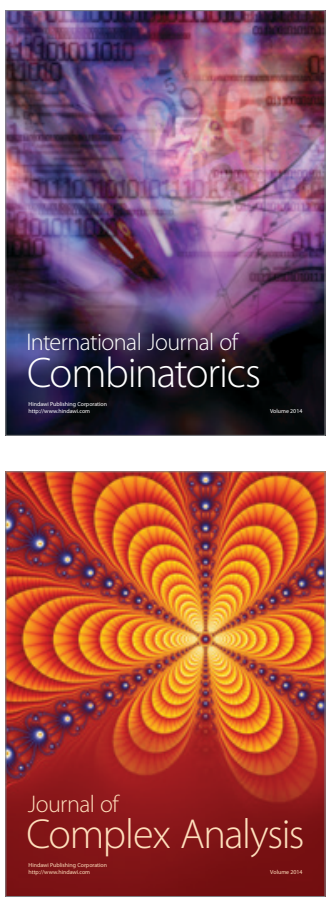

International Journal of

Mathematics and

Mathematical

Sciences
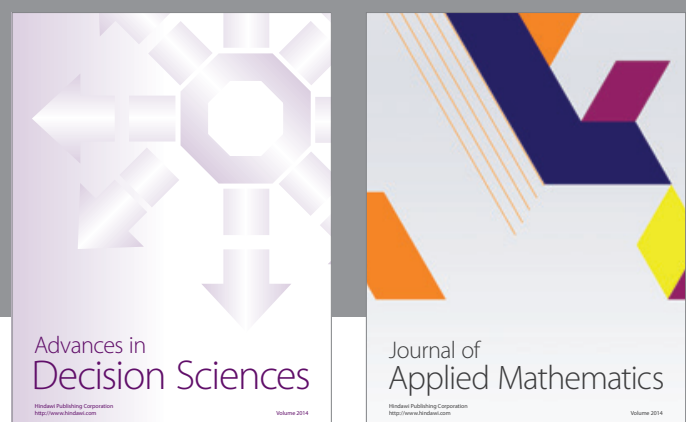

Journal of

Applied Mathematics
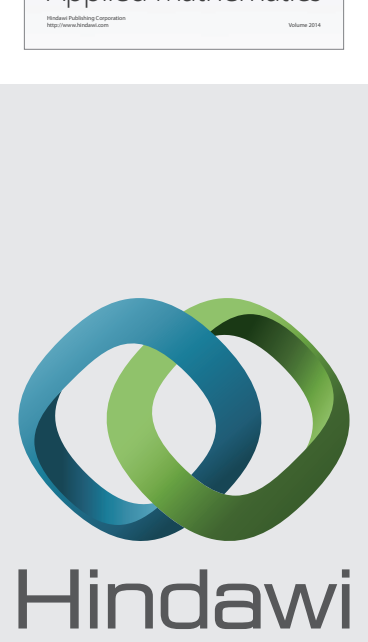

Submit your manuscripts at http://www.hindawi.com
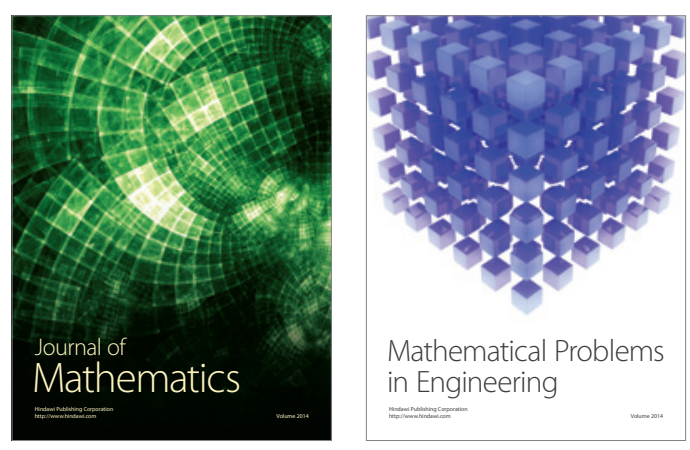

Mathematical Problems in Engineering
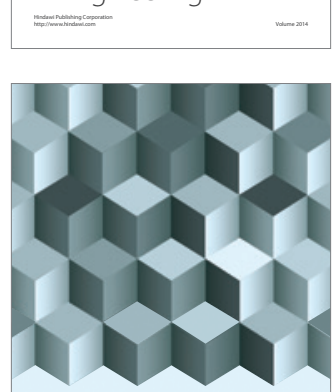

Journal of

Function Spaces
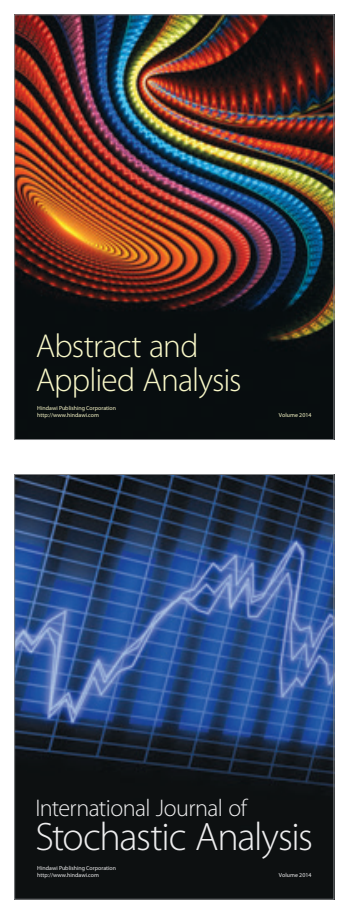

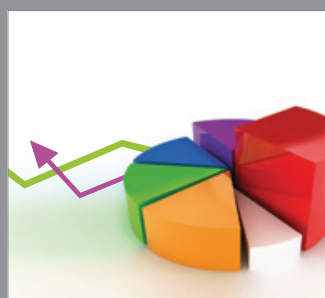

ournal of

Probability and Statistics

Promensencen
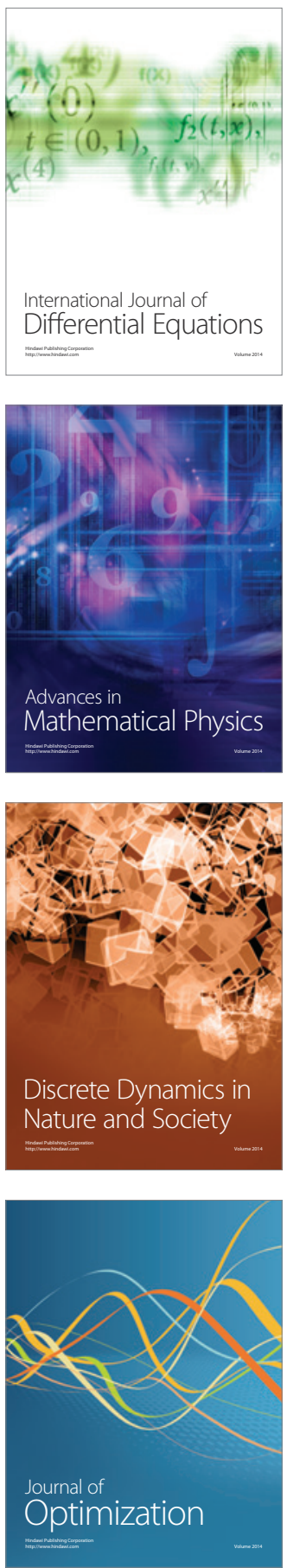